\title{
Treatment Patterns and Health Care Costs of Lupus Nephritis in a United States Payer Population
}

This article was published in the following Dove Press journal:

Open Access Rheumatology: Research and Reviews

\author{
Laura Bartels-Peculis (D) \\ Ajay Sharma ${ }^{2}$ \\ Alison M Edwards ${ }^{2}$ \\ Anirudh Sanyal ${ }^{2}$ \\ Erin Connolly-Strong' \\ Winnie W Nelson (ID)
}

'Mallinckrodt Pharmaceuticals, Inc., Bedminster, NJ, USA; ${ }^{2}$ Healthagen LLC, New York, NY 10017, USA
Correspondence: Laura Bartels-Peculis Email laura.bartels@mnk.com

\begin{abstract}
Objective: To describe the characteristics, treatment patterns, health care resource utilization (HCRU), and cost of care for members of a large United States (US) health insurance plan with lupus nephritis (LN).
\end{abstract}

Methods: A retrospective observational study was conducted using a health insurance plan database to identify adult members with a diagnosis of LN. Medical and pharmacy claims were used to describe demographics, comorbidities, HCRU, and cost patterns over a 12month follow-up period for each patient, between January 1, 2014, and December 31, 2016. All study variables were examined descriptively.

Results: A total of 1039 patients were available for analysis (median age, 47 years; 83\% female). The median Charlson Comorbidity Index (CCI) was 3.3. Less than half (41\%) of patients received immunosuppressive therapies commonly used to treat LN. Evidence indicated that $58 \%$ of the study population were prescribed corticosteroid therapy, in most cases (73\%) for more than 60 days. Adverse events known to be associated with corticosteroid therapy were recorded in $58 \%$ of patients. Guideline-recommended preventive therapy with hydroxychloroquine was prescribed for $54 \%$ of members with LN. Nearly half $(47 \%)$ of members with LN did not see a nephrologist and more than one-third (36\%) did not see a rheumatologist over 1 year of follow-up. Rates of all-cause hospitalization and emergency department (ED) use were $25 \%$ and $35 \%$, respectively. The mean all-cause per-member-permonth (PMPM) medical cost for the study population was \$2801, with LN-specific costs accounting for \$1147 PMPM.

Conclusion: Patients with LN who are insured through a large US health plan appeared to underutilize outpatient specialist services and guideline-recommended hydroxychloroquine therapy. Corticosteroid use and adverse events known to be associated with corticosteroids were common in this cohort.

Keywords: lupus nephritis, provider visits, health care resource utilization, health care costs, real-world evidence

\section{Introduction}

Lupus nephritis (LN), a common complication of systemic lupus erythematosus (SLE), results from the loss of self-tolerance, production of autoantibodies, and intrarenal inflammation. ${ }^{1}$ An estimated $40 \%$ to $60 \%$ of patients with SLE develop LN, with nephritis sometimes present at the diagnosis of SLE. ${ }^{2-4}$ Risk factors for LN include younger age (including SLE diagnosis before adulthood), ${ }^{5}$ male gender, and non-white race. ${ }^{2,3}$

Like SLE, LN features alternating periods of active and quiescent disease. Immunosuppressive therapy may prevent disease flares, but inflammation can permanently damage renal cells if untreated or unresponsive to treatment. ${ }^{6}$ 
About $10 \%$ of patients with LN progress to end-stage renal disease within 10 years. $^{2} \mathrm{LN}$ raises the alreadyhigh mortality rates ${ }^{2}$ and health care $\operatorname{costs}^{7,8}$ associated with SLE. ${ }^{8}$

Only a few studies document treatment patterns, health care resource utilization (HCRU), and medical costs among patients with LN insured by payers in the United States. ${ }^{7-9}$ An investigation comparing medication use in patients with LN from 2000 to 2006 against recommendations that were not released until 3 and 6 years later (2009 and 2012) concluded that prescribing did not adhere to the subsequent recommendations. ${ }^{10-13}$

This paper reports the characteristics, treatment patterns, and cost of care for adults with LN covered by Aetna, a large health plan based in the United States. The treatment patterns are discussed in light of the 2012 American College of Rheumatology (ACR) and European League Against Rheumatism guidelines for managing lupus nephritis. ${ }^{12,13}$

\section{Patients and Methods}

\section{Study Design and Data Source}

The Aetna database contains information from an integrated health plan with both medical and pharmacy benefits. It covers members in all 50 states in the US and Washington, DC, and lists enrollment status, demographics (eg, age, sex, and region), International Classification of Diseases (ICD)-9 and ICD-10 diagnosis codes, procedural codes, and outpatient and inpatient visits as well as pharmacy claims utilizing National Drug Code and laboratory data associated with Logical Observation Identifiers Names and Codes. It links claims and laboratory results to individuals and their respective providers. The study protocol was reviewed and approved by the Aetna Institutional Review Board.

For the time period evaluated in this study (January 1, 2014 to December 31, 2016), Aetna's database covers approximately 10 million commercial members and 2.4 million Medicare Advantage members. The database includes information about members in all 50 states including Washington, DC, and is considered representative of the US population.

Medical conditions of interest were identified through Aetna's proprietary Health Profile Database (HPD), ${ }^{14}$ which uses medical and pharmacy claims and laboratory data to classify individuals having approximately 90 conditions. This study was approved by an external LN Institutional Review Board.

\section{Study Population}

Inclusion criteria required that members have at least one medical claim with a diagnosis code for LN (using the ICD-9 or ICD-10), at least 18 years of age on the date of their first LNrelated claim (ie, the index date), with 6 months of continuous medical and pharmacy coverage prior to (baseline period) and at least 12 months following (follow-up period) the index date.

Members with 1 or more claims for clinician- or facilityprovided services for both SLE and nephritis or 1 or more claims for LN (codes in Supplementary Table 1) during the study period were considered diagnosed with LN. Exclusion criteria included members who were receiving end-of-life care. The Charlson Comorbidity Index (CCI), a 10-year predictor of mortality, was calculated to describe disease burden in this population. ${ }^{15}$ Patient clinical characteristics and demographics were assessed in the baseline period.

\section{Statistical Methodology}

Diagnoses and comorbidities, prescription drug fills and inhospital drug usage, HCRU, and cost in various settings (emergency department [ED], inpatient, and outpatient) were derived from medical or pharmacy claims. LN-specific utilization was defined as an event with a diagnosis of $\mathrm{LN}$ in any diagnostic position. We defined drug exposure as at least one fill or claim for any drug in a medication class of interest or for a specific drug of interest. Medications of interest were corticosteroids, six immunosuppressive agents commonly prescribed for LN (ie, azathioprine, cyclophosphamide, cyclosporine, mycophenolate, rituximab, and tacrolimus), and adjunctive therapies recommended by the ACR guidelines for LN (ie, statins, hydroxychloroquine, and renin-angiotensin system blockade). ${ }^{12}$ Provider specialty was determined by linking the provider identification number on a medical claim to a provider database listing the specialty. We captured patient demographic information utilizing the health plan's membership file. Data reflect 12 months of follow-up for each patient during the 3-year study period (January 1, 2014, through December 31, 2016).

All study variables were examined descriptively. Continuous variables were characterized as mean, standard deviation, median, range, and interquartile range. Categorical variables were reported as counts and percentages. All-cause and LN-specific HCRU and cost were calculated using allowed medical and pharmacy data (drugs administered and dispensed), and allowable costs of health care services. Average costs were based on 2017 data and were additionally summarized as per-member-per-month (PMPM). 


\section{Patient and Public Involvement}

This was a retrospective claims data analysis, and neither patients nor the public were involved in the design and execution of this study.

\section{Results}

\section{Study Population Demographics and Comorbidities}

A total of 1039 members (Table 1) met the inclusion criteria after eliminating those with claims for end of life care $(n=43)$, age

Table I Study Population Characteristics

\begin{tabular}{|c|c|}
\hline Total Study Population $(\mathbf{N}=1039)$ & n (\%)* \\
\hline Age (median, IQR) & 47 years ( $35-57$ years) \\
\hline Female & $866(83)$ \\
\hline \multicolumn{2}{|l|}{ Insurance type } \\
\hline Commercial & $833(80)$ \\
\hline Medicare Advantage & $206(20)$ \\
\hline Income (median, IQR) & $\begin{array}{l}\$ 63,891(\$ 48,882- \\
\$ 84,035)\end{array}$ \\
\hline \multicolumn{2}{|l|}{ Residential setting } \\
\hline Urban & $984(95)$ \\
\hline Rural & $50(5)$ \\
\hline \multicolumn{2}{|l|}{ Region } \\
\hline Northeast & $376(36)$ \\
\hline Midwest & $187(18)$ \\
\hline South & $347(33)$ \\
\hline West & $124(12)$ \\
\hline Missing & $5(<1)$ \\
\hline \multicolumn{2}{|l|}{ Comorbidities (n (\%) } \\
\hline Hypertension & $705(68)$ \\
\hline Hyperlipidemia & $442(43)$ \\
\hline Chronic renal failure & $432(42)$ \\
\hline Nonspecific gastritis/dyspepsia & $368(35)$ \\
\hline Low back pain & $240(23)$ \\
\hline Chronic thyroid disorders & $229(22)$ \\
\hline Diabetes mellitus & $185(18)$ \\
\hline Depression & $177(17)$ \\
\hline Osteoarthritis & $173(17)$ \\
\hline Obesity & $158(15)$ \\
\hline \multicolumn{2}{|l|}{ Charlson Comorbidity Index } \\
\hline Mean (SD) & $3.325(2.5)$ \\
\hline 0 & $10(I)$ \\
\hline I & $295(28)$ \\
\hline $2-3$ & 357 (34) \\
\hline $4-5$ & $217(21)$ \\
\hline $6+$ & $160(15)$ \\
\hline
\end{tabular}

Note: *Unless otherwise noted.

Abbreviations: IQR, interquartile range; SD, standard deviation. younger than $18(n=42)$, and less than 12 months of continuous follow-up eligibility $(n=1186)$. The median age of study population was 47 years. Most study participants were female (83\%), urban (95\%), based in the Northeast and South (36\% and 33\%, respectively), and covered by commercial insurance ( $80 \%)$. The mean CCI was 3.3. Hypertension, hyperlipidemia, and chronic renal failure were the most common comorbidities.

\section{Medication Use}

Table 2 lists the proportions of patients with at least 1 pharmacy claim for any medication of interest during the 12-month follow-up period. About $10 \%$ of patients had evidence of receiving immunosuppressive therapy without corticosteroids while $31 \%$ had claims for both immunosuppressive therapy and a corticosteroid. One-third (33\%) had no evidence of receiving immunosuppressive or corticosteroid therapy. Mycophenolate was the most commonly prescribed immunosuppressive agent.

\section{Immunosuppressive Therapies}

Figure 1 summarizes use of immunosuppressive therapy commonly prescribed for SLE in our study population. Of the $41 \%$ (421/1039) who received any immunosuppressive therapy, most $(77 \%$; 325/421) received only 1 of the 6 medications of interest. Most patients who received any immunosuppressive therapy also received corticosteroids $(76 \% ; 322 / 421)$.

Table 2 Medication Use*

\begin{tabular}{|l|l|l|}
\hline Medication/Medication Class & $\mathbf{n}$ & \% of N = I039 \\
\hline Guideline-Recommended Therapies & $\dagger$ & \\
Statins/antihyperlipidemics & 286 & $27.5 \%$ \\
Hydroxychloroquine & 559 & $53.8 \%$ \\
ACEls/ARBs & 467 & $45.0 \%$ \\
\hline Immunosuppressive Medications & & \\
Mycophenolate mofetil & 308 & $29.6 \%$ \\
Azathioprine & 99 & $9.5 \%$ \\
Tacrolimus & 54 & $5.2 \%$ \\
Cyclosporine & 40 & $3.8 \%$ \\
Rituximab & 18 & $1.7 \%$ \\
Cyclophosphamide & 6 & $0.6 \%$ \\
\hline Corticosteroids & & \\
Methylprednisolone & 109 & $10.5 \%$ \\
Prednisone & 552 & $53.1 \%$ \\
\hline
\end{tabular}

Notes: *Percentages and raw data do not sum to $100 \%$ or totals as some patients received more than I medication. ${ }^{\dagger} A C R$ guidelines for $L N$ recommend statin therapy for patients with low-density lipoprotein cholesterol (LDL-C) $>100 \mathrm{mg} / \mathrm{dL}$ (2.586 mmol/L), hydroxychloroquine for all patients with $L N$, and ACEls/ARB therapy for patients with proteinuria $(\geq 0.5 \mathrm{~g}$ per 24 hours or equivalent by protein/creatinine ratios on spot urine samples). ${ }^{12}$

Abbreviations: ACEls, angiotensin-converting enzyme inhibitors; ARBs, angiotensin receptor blockers. 


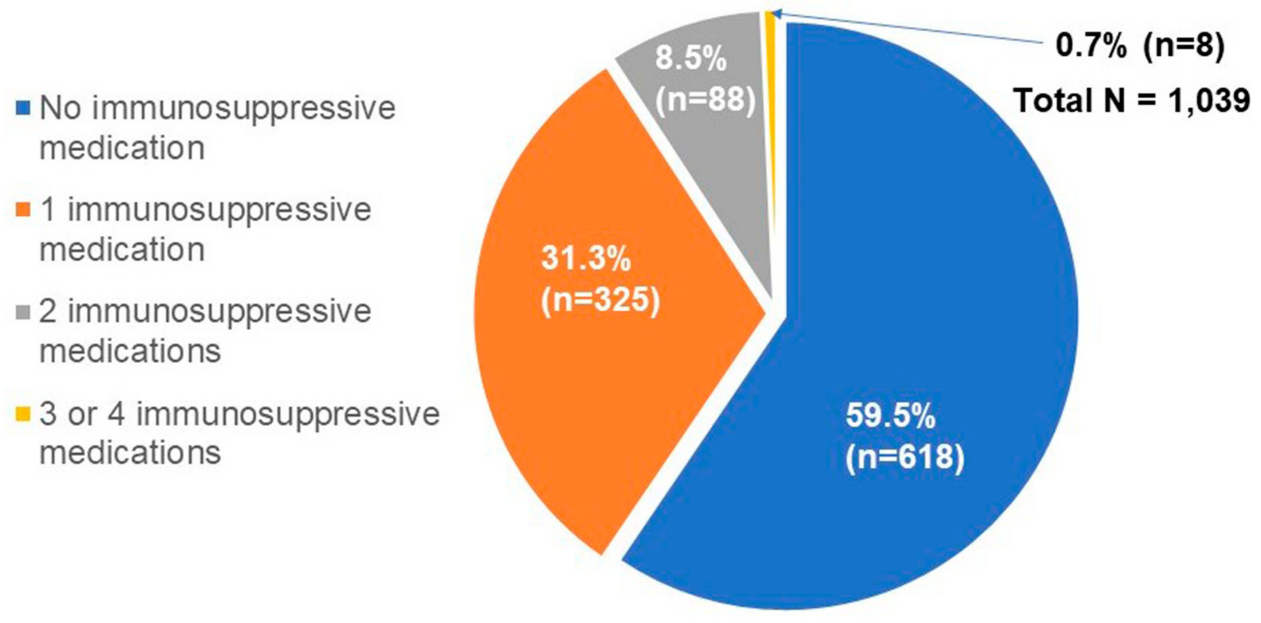

Figure I Use of immunosuppressive medications over 12 months.

\section{Corticosteroid Exposure}

More than half $(58 \% ; 602)$ of the study population received corticosteroids. Most of these patients $(73 \%$; 438/602) received more than 60 days' supply (Supplementary Figure 1). Almost one-third (29\%; 173/ 602) received steroids for more than 300 days.

There were 606 instances of medical claims during the follow-up period for adverse events known to be associated with corticosteroids. These events included osteoporosis $(3.6 \%)$, fracture $(8.8 \%)$, glaucoma $(1 \%)$, secondary diabetes mellitus (4\%), myocardial infarction (MI; 6\%), gastrointestinal (GI) ulcer or GI bleed (7\%), and avascular necrosis (6\%).

\section{Adherence to Guideline-Recommended Adjunctive Therapies}

The most recent ACR guidelines for the care of $\mathrm{LN}$ recommend hydroxychloroquine for all patients, reninangiotensin system blockade (angiotensin-converting enzyme inhibitors [ACEIs] or angiotensin II receptor blockers $[\mathrm{ARBs}])$ for those with proteinuria $(\geq 0.5 \mathrm{~g}$ per 24 hours or equivalent by protein/creatinine ratios on spot urine samples), and statin therapy for patients with lowdensity lipoprotein cholesterol (LDL-C) $>100 \mathrm{mg} / \mathrm{dL}$ $(>2.586 \mathrm{mmol} / \mathrm{L})(12)$. More than half of the study population $(54 \%)$ had evidence of therapy with hydroxychloroquine, $45 \%$ had evidence of an ACEI/ARB treatment, and $28 \%$ received statin therapy.

\section{Health Care Resource Utilization}

Provider Visits by Specialty

During the study's 1-year follow-up period, $47 \%$ of members with LN did not visit a nephrologist and 36\% did not visit a rheumatologist (Table 3 ). Among patients who had any visits during the follow-up year, most had between 1 and 4 visits (Supplementary Table 2).

\section{Hospitalization and ED Visits}

A quarter of the study population was hospitalized at least once during the 12-month follow-up period. Most of these patients ( $20 \%$ of the study population) had 1 or 2 inpatient visits but $5 \%$ of the study population had 3 or more inpatient visits. More than a third of the study population (35\%) had evidence of at least 1 ED visit during followup. Most of those ( $27 \%$ of the study population) had 1 or 2 ED visits but $8 \%$ of the study had 3 or more ED visits.

Table 3 Frequency of Member Visits to Providers Over 12 Months, by Specialty (Member $\mathrm{n}[\%]$ ).*

\begin{tabular}{|c|c|c|c|}
\hline & 0 Visits & $\geq \mathbf{I}$ Visits & $\begin{array}{l}\text { Average } \\
\text { Number of } \\
\text { Visits Among } \\
\text { Members with } \\
\text { Any Visits }\end{array}$ \\
\hline Cardiology & $634(61)$ & 405 (39) & 4.7 \\
\hline Family practice & $564(54)$ & $475(46)$ & 4.8 \\
\hline Hematology/oncology & $875(84)$ & $164(16)$ & 7.3 \\
\hline Infectious diseases & $956(92)$ & $83(8)$ & 8.8 \\
\hline Internal medicine & $392(38)$ & $647(62)$ & 8.4 \\
\hline Nephrology & 491 (47) & $548(53)$ & 5.9 \\
\hline Obstetrics/gynecology & $680(65)$ & $359(35)$ & 2.8 \\
\hline Ophthalmology & $664(64)$ & $375(36)$ & 2.1 \\
\hline Pulmonary/critical care & $900(87)$ & $139(14)$ & 5.5 \\
\hline Rheumatology & $379(36)$ & $660(64)$ & 4.8 \\
\hline
\end{tabular}

Notes: *Specialties are listed alphabetically. Total $\mathrm{N}=1039$. Some percentages do not add up to 100 due to rounding. 


\section{Health Care Cost}

Annual mean (SD) and PMPM costs were calculated separately for all-cause medical (inpatient care, outpatient procedures, ED, professional office, and other) and pharmacy costs and for LN-specific medical costs. Mean allcause medical costs for the study population were $\$ 33,620$ $(\$ 95,568)$, with a PMPM cost of $\$ 2801$. Mean LN-specific medical costs were $\$ 13,758(\$ 39,889)$, with a PMPM cost of 1147. Medical costs specific to LN accounted for about $41 \%$ of the total medical costs for the study population. Mean all-cause pharmacy costs for the study population were $\$ 11,847(\$ 17,666)$, with a PMPM of $\$ 987$.

The total mean, all-cause health care costs per patient per year for the study population, summing medical and pharmacy costs, was $\$ 45,469$. Figure 2 shows the percentages of this cost accounted for by six categories: inpatient care, pharmacy, outpatient procedures, ED, professional office, and other.

\section{Discussion}

This retrospective analysis of data from a large health plan describes the characteristics, treatment patterns, HCRU, and cost of care for a group of patients with $\mathrm{LN}$ in the US. The high rates of hypertension, hyperlipidemia, and chronic renal failure in this population $(68 \%, 43 \%$, and $42 \%$, respectively) were especially striking given the cohort's median age of 47 years old. For comparison, the prevalence of hypertension in US adults is $29 \%$ overall and $33.2 \%$ among those 40 to 59 years old. ${ }^{16}$ About $12 \%$ of US adults have elevated levels of total cholesterol, with rates of $17 \%$ and $18 \%$ for men and women (respectively) ages 40 to 59 years old. ${ }^{17}$ Crude prevalence of chronic kidney disease is $12 \%$ to $15 \%$ in the US population, and $9.2 \%$ in people ages 40 to 59 years old. ${ }^{18}$
Our population appeared to have a relatively high comorbidity burden compared to others with LN, with a mean CCI of 3.3; a retrospective claims data analysis of 1259 incident cases with $\mathrm{LN}$ reported a mean CCI of 1.8. ${ }^{19}$

Findings suggest that patients with LN underutilize guideline-recommended hydroxychloroquine as well as outpatient specialist services. More than one-third (36\%) of patients with LN did not see a rheumatologist and nearly half (47\%) did not see a nephrologist during the 12month follow-up period.

ACR guidelines recommend that all patients with LN should receive hydroxychloroquine in the absence of a contraindication; all patients with $\mathrm{LN}$ and proteinuria $\geq 0.5 \mathrm{~g} / 24$ hours should receive ACEI or ARB therapy; and patients with LN and LDL-C $>100 \mathrm{mg} / \mathrm{dL}$ should receive statin therapy. ${ }^{12}$ A little more than half (54\%) of our study cohort had evidence of receiving hydroxychloroquine therapy. The proportion of members who met the proteinuria and LDL-C criteria for guideline-recommended ACEI/ARB or statin therapy, respectively, could not be extracted from the claims data available to the authors.

About $41 \%$ of our study population had a prescription for immunosuppressive therapy. According to the 2012 ACR guidelines, immunosuppressive therapy is recommended for induction and maintenance therapy in LN patients with class III disease or higher. ${ }^{12}$ Claims data available to us included renal biopsy findings ${ }^{20}$ and so did not allow determination of the proportion of members who met these criteria for immunosuppressive therapy.

More than half of the study cohort was prescribed corticosteroids at some point during the follow-up period. Corticosteroids are recommended for some cases of class II LN and may be considered in class I disease, according

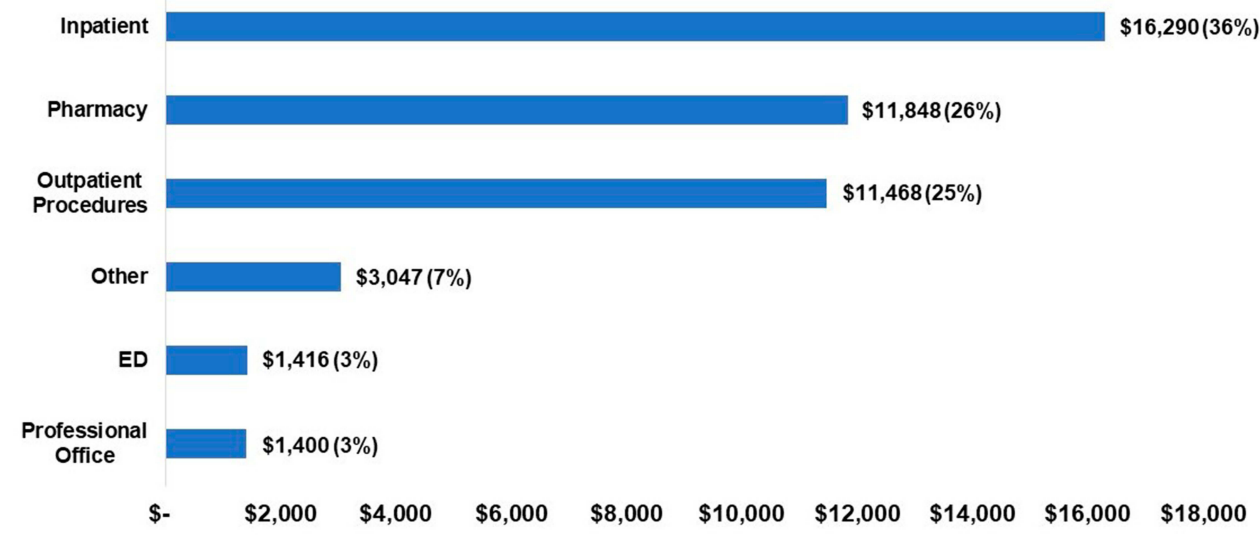

Figure 2 Mean all-cause annual health care cost per member per year with lupus nephritis. 
to the European guidelines. ${ }^{13}$ Steroids are part of recommended induction (3-6 months) and maintenance therapy for patients with LN class III and higher. ${ }^{12,13}$ Without renal biopsy information, we cannot determine whether corticosteroid use in this cohort was consistent with the guidelines.

Other studies have reported higher US health care costs for patients with $\mathrm{LN}$ compared with controls ${ }^{7,8}$ with the broader group of patients with $\mathrm{SLE}^{8}$ or with SLE and no nephritis. ${ }^{9}$ Mean annual health care costs for LN in our study were similar to those reported by Furst and colleagues $\left(\$ 33,472\right.$ in 2009 US dollars). ${ }^{7}$ Pharmacy claims accounted for a much higher share of the cost in our study $(26 \%)$ than in these investigations $\left(12.5 \%,{ }^{7} 10 \%\right.$ $15 \%,{ }^{8}$ and $20 \%{ }^{9}$ ), perhaps reflecting the increasing adoption of biologic agents in SLE.

The treatment costs of corticosteroid use are well documented. One report found that, among patients receiving corticosteroids for at least 60 days, the costs of managing steroid-associated adverse events exceeded disease-related medical costs ( $\sim 40 \%$ and $\sim 25 \%$, respectively). Cost and health care resource utilization generally rose with dosage category. $^{21}$ These findings come from a claims database study of adults with various autoimmune or inflammatory conditions. ${ }^{21}$ Database studies in the United States that are specific to SLE also have linked corticosteroid use and higher corticosteroid dose with higher health care utilization and costs. ${ }^{22,23}$

More than half $(58 \%)$ of patients in our study cohort had claims for adverse events known to be associated with corticosteroid exposure. The same proportion of the population had corticosteroid exposure; $73 \%$ of those prescribed corticosteroid therapy received it for more than 60 days. The claims data analyzed do not provide sufficient evidence to determine whether plan members who experienced AEs known to be linked to steroid toxicity were the same members with prescriptions for corticosteroid therapy. Toxicity could have developed following corticosteroid therapy that predated the study period, for example. Still, both corticosteroid use and events known to be linked to corticosteroid toxicity are common in our population.

Other studies demonstrate that even short-term corticosteroid therapy can lead to increased risk of adverse events. Using oral corticosteroids for less than 30 days has been associated with significantly increased rates of fracture, sepsis, and venous thromboembolism compared with the period before the oral steroid was prescribed, in a US-based population-based cohort study. ${ }^{24}$ An increased risk of MI after less than 30 days of oral corticosteroid therapy was reported in a UK-based case-control study. ${ }^{25}$

Corticosteroid exposure was associated with increased risk and earlier emergence of adverse events in a database study of privately insured adults in the United States. ${ }^{26}$ The rate of adverse events rose with dose category, but even those receiving low-dose steroids for $>60$ days had significantly higher risk of certain adverse events (sepsis, pneumonia, and MI) compared with those taking steroids intermittently. ${ }^{26}$ Corticosteroid use in patients with SLE has been associated with increased risk of hypertension, ${ }^{27}$ which affected $68 \%$ of our study group. Some of the side effects known to be associated with corticosteroids observed in our patient population (eg, fracture, MI, or GI bleed), might have contributed to the inpatient hospitalization rate $(25 \%$ of patients) and the use of the ED (35\% of patients).

The number of patients with claims for corticosteroidassociated events during the follow-up period slightly exceeds the number of patients who received corticosteroids during the follow-up period. Adverse events might have stemmed from corticosteroid treatment that predated the 12-month follow-up period.

Our study has several limitations beyond those mentioned. The relatively short follow-up period provides a cross-sectional view of LN patients with a lifelong illness that flares and remits over time. Secondary use of health plan data carries the limitation of potential coding and billing inaccuracies. For example, our population may have included patients with diffuse proliferative nephritis - the most severe form of lupus nephritis - if providers did not code separately for this condition. While informative for other commercial payers, our data may not be generalizable to Medicare or Medicaid beneficiaries or uninsured patients. However, the magnitude of some of our findings is consistent with those of prior studies, suggesting that any inaccuracies may not have significant impact.

Future research with a longitudinal view can study the progression of the disease over time and the impact of therapies. Access to renal biopsy results and laboratory data could enable determination of LN class and rates of proteinuria and elevated LDL-C, facilitating a more detailed assessment of the proportion of patients not receiving guideline-recommended therapy.

In summary, patients with LN in a commercial health plan appeared to underutilize guideline-recommended 
therapies as well as key outpatient care. Underuse of recommended care as well as extensive exposure to steroids may drive hospitalization and ED visits. A high rate of corticosteroid exposure and of adverse events known to be associated with corticosteroid therapy was observed in this population, as would be expected in patients with LN. Adherence to guideline-recommended therapy and minimizing use of steroids may improve care, reduce adverse events, and lower cost.

\section{Acknowledgments}

Eileen McCaffrey, MA, of Global Outcomes Group provided writing support and Esther Tazartes, MS, of Global Outcomes Group provided editorial support. Financial support for this manuscript was provided by Mallinckrodt Pharmaceuticals, Inc.

\section{Disclosure}

Laura Bartels-Peculis, PharmD, is an employee of Mallinckrodt Pharmaceuticals. Ajay Sharma DO, is an employee of Healthagen LLC. Alison M. Edwards, MStat, is a data consultant at Aetna, Inc. Anirudh Sanyal, B. Eng., is a senior informatics analyst at Aetna, Inc. Erin Connolly-Strong, $\mathrm{PhD}$, is an employee of Mallinckrodt Pharmaceuticals and owns stock in Mallinckrodt Pharmaceuticals. At the time of design, implementation, and manuscript drafting, Winnie W. Nelson, PharmD, MS, MBA, was an employee of Mallinckrodt Pharmaceuticals. Winnie W. Nelson owns stock in Mallinckrodt Pharmaceuticals. The authors report no other conflicts of interest in this work.

\section{References}

1. Liu Y, Anders HJ. Lupus nephritis: from pathogenesis to targets for biologic treatment. Nephron Clin Pract. 2014;128(3-4):224-231. doi: $10.1159 / 000368581$

2. Hanly JG, O'Keeffe AG, Su L, et al. The frequency and outcome of lupus nephritis: results from an international inception cohort study. Rheumatology (Oxford, England). 2016;55(2):252-262. doi:10.1093/ rheumatology/kev311

3. Almaani S, Meara A, Rovin BH. Update on lupus nephritis. Clin J Am Soc Nephrol. 2017;12(5):825-835. doi:10.2215/CJN.05780616

4. Barber MRW, Hanly JG, Su L, et al. Economic evaluation of lupus nephritis in the systemic lupus international collaborating clinics inception cohort using a multistate model approach. Arthritis Care Res (Hoboken). 2018;70(9):1294-1302. doi:10.1002/acr.23480

5. Tucker LB, Uribe AG, Fernandez M, et al. Adolescent onset of lupus results in more aggressive disease and worse outcomes: results of a nested matched case-control study within LUMINA, a multiethnic US cohort (LUMINA LVII). Lupus. 2008;17(4):314-322. doi:10.11 77/0961203307087875
6. Chan TM. Treatment of severe lupus nephritis: the new horizon. Nat Rev Nephrol. 2015;11(1):46-61. doi:10.1038/nrneph.2014.215

7. Furst DE, Clarke A, Fernandes AW, et al. Medical costs and healthcare resource use in patients with lupus nephritis and neuropsychiatric lupus in an insured population. J Med Econ. 2013;16(4):500-509. doi:10.3111/13696998.2013.772058

8. Li T, Carls GS, Panopalis P, Wang S, Gibson TB, Goetzel RZ. Longterm medical costs and resource utilization in systemic lupus erythematosus and lupus nephritis: a five-year analysis of a large medicaid population. Arthritis Rheum. 2009;61(6):755-763. doi:10.1002/art.24 545

9. Pelletier EM, Ogale S, Yu E, Brunetta P, Garg J. Economic outcomes in patients diagnosed with systemic lupus erythematosus with versus without nephritis: results from an analysis of data from a US claims database. Clin Ther. 2009;31(11):2653-2664. doi:10.1016/j.clinthera. 2009.11.032

10. Yazdany J, Feldman $\mathrm{CH}$, Liu J, Ward MM, Fischer MA, Costenbader KH. Quality of care for incident lupus nephritis among medicaid beneficiaries in the United States. Arthritis Care Res (Hoboken). 2014;66(4):617-624. doi:10.1002/acr.22182

11. Yazdany J, Panopalis P, Gillis JZ, et al. A quality indicator set for systemic lupus erythematosus. Arthritis Rheum. 2009;61(3):370-377. doi:10.1002/art.24356

12. Hahn BH, McMahon M, Wilkinson A, et al. American college of rheumatology guidelines for screening, case definition, treatment and management of lupus nephritis. Arthritis Care Res (Hoboken). 2012;64(6):797-808. doi:10.1002/acr.21664

13. Bertsias GK, Tektonidou M, Amoura Z, et al. Joint European league against rheumatism and European renal association-european dialysis and transplant association (EULAR/ERA-EDTA) recommendations for the management of adult and paediatric lupus nephritis. Ann Rheum Dis. 2012;71(11):1771-1782. doi:10.1136/annrheumdis-20 12-201940

14. Hanchak NA, Murray JF, Hirsch A, McDermott PD, Schlackman N. USQA health profile database as a tool for health plan quality improvement. Manag Care Q. 1996;4(2):58-69.

15. Charlson M, Szatrowski TP, Peterson J, Gold J. Validation of a combined comorbidity index. J Clin Epidemiol. 1994;47(11):12 45-1251. doi:10.1016/0895-4356(94)90129-5

16. Fryar CD, Ostchega Y, Hales CM, Zhang G, Kruszon-Moran D. Hypertension Prevalence and Control Among Adults: United States, 2015-2016. NCHS Data Brief, No 289. Hyattsville, MD: National Center for Health Statistics; 2017.

17. Carroll MD, Fryar CD, Nguyen DT. High Total and Low HighDensity Lipoprotein Cholesterol in Adults: United States, 2015-2016. NCHS Data Brief, No 290. Hyattsville, MD: National Center for Health Statistics; 2017.

18. Centers for Disease Control and Prevention. Chronic kidney disease surveillance system-United States. Available from: http://www.cdc. gov/ckd (http://www.cdc.gov/ckd). Accessed February 6, 2019.

19. Xie L, Kariburyo F, Sah J, Lofand J, Li N. Real world medication use in incident systemic lupus erythematosus and lupus nephritis patients [abstract]. Arthritis Rheumatol. 2018;70(suppl 10).

20. Weening JJ, D'Agati VD, Schwartz MM, et al. The classification of glomerulonephritis in systemic lupus erythematosus revisited. $J \mathrm{Am}$ Soc Nephrol. 2004;15(2):241-250. doi:10.1097/01.ASN.0000108969. 21691.5D

21. Rice JB, White AG, Johnson M, et al. Healthcare resource use and cost associated with varying dosages of extended corticosteroid exposure in a US population. J Med Econ. 2018;21(9):846-852. doi:10.1080/13696998.2018.1474750

22. Chen SY, Choi CB, Li Q, et al. Glucocorticoid use in patients with systemic lupus erythematosus: association between dose and health care utilization and costs. Arthritis Care Res (Hoboken). 2015;67 (8):1086-1094. doi:10.1002/acr.22574 
23. Kabadi S, Yeaw J, Bacani AK, et al. Healthcare resource utilization and costs associated with long-term corticosteroid exposure in patients with systemic lupus erythematosus. Lupus. 2018;27 (11):1799-1809. doi:10.1177/0961203318790675

24. Waljee AK, Rogers MA, Lin P, et al. Short term use of oral corticosteroids and related harms among adults in the United States: population based cohort study. BMJ. 2017;357:j1415. doi:10.1136/ bmj.j1415

25. Varas-Lorenzo C, Rodriguez LA, Maguire A, Castellsague J, PerezGutthann S. Use of oral corticosteroids and the risk of acute myocardial infarction. Atherosclerosis. 2007;192(2):376-383. doi:10.10 16/j.atherosclerosis.2006.05.019
26. Rice JB, White AG, Johnson M, et al. Quantitative characterization of the relationship between levels of extended corticosteroid use and related adverse events in a US population. Curr Med Res Opin. 2018;34(8):1519-1527. doi:10.1080/03007995.2018.1474090

27. Shah M, Chaudhari S, McLaughlin TP, et al. Cumulative burden of oral corticosteroid adverse effects and the economic implications of corticosteroid use in patients with systemic lupus erythematosus. Clin Ther. 2013;35(4):486-497. doi:10.1016/j.clinthera.2013.03.001

\section{Publish your work in this journal}

Open Access Rheumatology Research and Reviews is an international, peer-reviewed, open access journal publishing original research, reports, editorials, reviews and commentaries on all aspects of clinical and experimental rheumatology in the clinic and laboratory including the following topics: Pathology, pathophysiology of rheumatological diseases; Investigation, treatment and management of rheumatological diseases; Clinical trials and novel pharmacological approaches for the treatment of rheumatological disorders. The manuscript management system is completely online and includes a very quick and fair peer-review system, which is all easy to use. Visit http://www.dovepress.com/testimonials.php to read real quotes from published authors. 